\title{
Article \\ A Vibration Similarity Model of Converter Transformers and Its Verification Method
}

\author{
Hao Wang, Li Zhang *, Youliang Sun, Guan Wang and Liang Zou (D)
}

School of Electrical Engineering, Shandong University, Jinan 250061, China; 202120603@mail.sdu.edu.cn (H.W.); youliang.s@163.com (Y.S.); guanpallas@sdu.edu.cn (G.W.); zouliang@sdu.edu.cn (L.Z.)

* Correspondence: zhleee@sdu.edu.cn

\begin{abstract}
According to the vibration characteristics of converter transformers, considering the Maxwell equation, magnetostrictive effect, Lorentz force and structural mechanics, the similarity criterion suitable for converter transformers is deduced in this paper. Using the finite element simulation platform, the multi physical field coupling model of converter transformers is constructed, and the scale coefficient is 0.1 . The magnetic flux density distribution, stress distribution, shape variable and vibration characteristics of the model before and after the similarity are analyzed. The results show that the variation law of the model before and after the similarity conforms to the similarity criterion, and the correctness of the similarity criterion is verified. The converter transformer vibration similarity model and its verification method can effectively reduce the unnecessary waste of resources before the preparation of converter transformers and have important reference value for the analysis and improvement of converter transformer vibration characteristics.
\end{abstract}

Keywords: converter transformer; vibration; similarity model; model verification

check for updates

Citation: Wang, H.; Zhang, L.; Sun, Y.; Wang, G.; Zou, L. A Vibration Similarity Model of Converter Transformers and Its Verification Method. Symmetry 2022, 14, 143. https://doi.org/10.3390/ sym14010143

Academic Editor: Angelo Freni

Received: 18 December 2021

Accepted: 4 January 2022

Published: 12 January 2022

Publisher's Note: MDPI stays neutral with regard to jurisdictional claims in published maps and institutional affiliations.

Copyright: (C) 2022 by the authors. Licensee MDPI, Basel, Switzerland. This article is an open access article distributed under the terms and conditions of the Creative Commons Attribution (CC BY) license (https:// creativecommons.org/licenses/by/ $4.0 /)$.

\section{Introduction}

In recent years, global energy and environmental problems have become increasingly prominent. In order to solve the problem of unbalanced distribution between energy base and load center, high voltage direct current (HVDC) transmission technology has developed rapidly because of its advantages of large transmission capacity, low transmission loss and no synchronous stability [1]. Converter transformers are one of the core pieces of equipment of the HVDC transmission system. It bears the joint action of alternating current $(\mathrm{AC})$ and direct current $(\mathrm{DC})$ voltage during operation. Compared with ordinary transformers, the load current of converter transformers contains a large amount of DC bias current and high-order harmonics, which makes its electromagnetic force and iron core resonance effect more obvious. In addition, the inherent magnetostrictive effect of iron core material under an alternating magnetic field, the vibration of the core and winding is very complex $[2,3]$. The vibration of the core and winding will be transmitted to the surface of the oil tank through the box structure, causing more complex vibration and noise of the whole converter transformer, which threatens the safe operation of the converter transformer. People have been committed to the research on the vibration generation mechanism and vibration suppression of converter transformers and are still troubled by its changeable test environment, complex electromagnetic field analysis and complex vibration propagation process. The main factors of the noise generation mechanism are still controversial, and the vibration suppression effect also faces difficulty in meeting the needs of the development of high voltage DC transmission technology [4-7].

According to the similarity principle, reducing the equal proportion of converter transformer to the volume that can be accommodated in the laboratory is an effective measure in solving the complex problem of the large capacity converter transformer test environment [8]. However, in many current studies, there is no unified similarity method for the research of converter transformer vibration suppression, and there is no effective 
method to verify the accuracy of converter transformer similarity criterion, which greatly reduces the reliability of the converter transformer scale model. Relevant studies mostly use the consistency of the electromagnetic field and vibration spectrum as the detection method for the correctness of the proportional model, but the premise is that the electromagnetic field and vibration signals of the model need to be analyzed after the physical model is prepared, and the model verification will lose its significance [9]. At the same time, due to the limitation of detection technology, it is difficult to analyze the stress of the internal components of the proportional model, so the means of verifying the vibration characteristics of the proportional model have been limited to the vibration signal analysis of the external points of the model [10]. Finite element simulation technology provides an idea for the analysis of such devices with complex internal structures and multi field stress coupling [11-14]. However, in the current finite element model research, electromagnetic field analysis is also used as the verification method of similar models, and the consistency of vibration characteristics before and after the similarity is not taken as the core idea of converter transformer vibration model verification. If the correctness of the similar model can be determined before the scale model is manufactured, the efficiency and reliability of the experiment will be greatly improved. Applying the idea of "point symmetry" to ensure that the response relationship between converter transformer input and vibration remains unchanged before and after the similarity, in this paper, the similarity criterion suitable for the study of the vibration characteristics of a converter transformer is derived, the converter transformer model and its similarity model are constructed, the magnetic flux density distribution, stress distribution, shape variable and vibration characteristics before and after the model similarity are analyzed, and the correctness of the similarity criterion is verified.

\section{Similarity Principle}

\subsection{Similarity of Electromagnetic Field Parameters}

According to the similarity theory, the similarity criterion can be derived from the differential equation describing the same physical process without solving the equation. It is more meaningful in theory and practice to select the controllable input under the principle of similarity. In this paper, the similarity criterion of converter transformers selects the current density $J$ as the reference value. On the one hand, the controllability of input voltage and current is considered; on the other hand, the loss is kept within the controllable range after the coil size is reduced. By introducing the scale factor $k$ of the similarity criterion, the length $l$, width $w$ and height $h$ of the commutation flow are reduced to $l^{\prime}=k l, w^{\prime}=k w, h^{\prime}=k h$, respectively, and the area $S$ is reduced to $S^{\prime}=k^{2} S$. The number of coil turns $N$ shall remain unchanged before and after the similarity, so the number of coil turns $n$ in unit length becomes $n^{\prime}=N / H^{\prime}=k^{-1} n$. The frequency is inversely proportional to the square of skin depth $\delta$ reduced to $\delta^{\prime}=k \delta$, therefore, the frequency of a converter transformer increases to $f^{\prime}=k^{-2} f$.

Based on the Maxwell equation and similarity theory, assuming that the current density $J$ remains unchanged, $I^{\prime} / S^{\prime}=I / S$, the current is reduced to $I^{\prime}=k^{2} I$. According to $U=I R$ and the similarity criteria of $I$ and $R$, the voltage is reduced to $U^{\prime}=k U$. According to $R=\rho l / S$, and the similarity criteria of $L$ and $S$, the resistance after the similarity becomes $R^{\prime}=\rho l^{\prime} / S^{\prime}=k^{-1} R, \rho$ is resistivity. According to the formula $G=I / U$, the conductivity after similarity $G^{\prime}=k^{2} I / k U=k G$. According to the relationship between capacitance and conductance $G / \sigma=C / \varepsilon$, get $C^{\prime}=k C$. Since $L=U / 2 \pi f I$, the inductance is reduced to $L^{\prime}=k L$ according to the similarity criteria of $U, I$ and $f$. The number of winding turns $N$ remains unchanged before and after the similarity, according to the integral form of Ampere's law $\int B d l=\mu_{0} N I$ knows that the magnetic induction intensity after the similarity $B^{\prime}=k B$. The similarity criteria of electromagnetic field parameters are listed in Table 1. 
Table 1. Similarity criterion of electromagnetic field parameters.

\begin{tabular}{cccccccc}
\hline & Length & Area & Frequency & Current & Voltage & Resistance & Magnetic Flux Density \\
\hline Original model & $l$ & $S$ & $f$ & $I$ & $U$ & $R$ & $B$ \\
Similarity model & $k l$ & $k^{2} S$ & $k^{-2} f$ & $k^{2} I$ & $k U$ & $k^{-1} R$ & $k B$ \\
\hline
\end{tabular}

\subsection{Similarity Analysis of Vibration Parameters}

In order to study and analyze the vibration of a converter transformer, it is necessary to start with the parameters directly related to the vibration and carry out similarity analysis on them to ensure that the vibration characteristics of the model are consistent before and after the similarity. According to previous studies, the vibration of a converter transformer is mainly divided into the vibration of iron core and winding. Therefore, in order to equivalent the vibration intensity and distribution of core column and winding, the mass matrix $M$ and stiffness coefficient matrix $K$ need to be reduced in equal proportion, so the natural frequency under the structure $\lambda=\sqrt{K / M}$ is almost unchanged, and the mode and vibration mode of the converter transformer are continued theoretically. Maintain the permeability, dielectric constant and conductivity of iron core and winding materials $\sigma$, permeability $\mu$ and resistivity $\rho$ consistent with the original model.

The finite element dynamic equation of winding and iron core structure is:

$$
M \ddot{s}(t)+C \dot{s}(t)+K s(t)=Q(t)
$$

where $\ddot{s}$ and $\dot{s}$ are the node acceleration vector and node velocity vector, $M$ is the mass matrix of the model, $C$ is the damping matrix of the model, $K$ is the stiffness matrix and $Q(t)$ is the load vector. Ignoring the influence of damping and external force, it can be further simplified as:

$$
\ddot{M}+\ddot{S}(t)=0
$$

This is the free vibration equation of the system, also known as the dynamic characteristic equation. From this equation, the natural frequency and natural vibration mode of the system can be solved.

Under the alternating magnetic field generated by alternating current, the winding will be subjected to radial electric force $F_{x}$ and axial electric force $F_{s}$ generated by axial and radial magnetic flux leakage:

$$
\begin{aligned}
& F_{x}=i B_{z t} \cdot 2 \pi r \\
& F_{z}=i B_{x t} \cdot 2 \pi r
\end{aligned}
$$

Then the resultant electromagnetic force $F_{l}$ of the two is:

$$
F_{l}=\sqrt{F_{x}^{2}+F_{z}^{2}}=i B_{t} \cdot 2 \pi r=2 \pi r k I^{2}(0.5+0.5 \cos 2 \omega t)
$$

where, $r$ is the winding radius, $i$ is the winding current, $B_{t}$ is the leakage flux density and $I$ is the effective value of the current. It can be seen that the force on the winding is related to the current $I$, the leakage magnetic field density $B_{t}$ and the winding radius $r$. After the similarity, $i^{\prime}=k^{2} i, B^{\prime}=k B, r^{\prime}=k r$, therefore, the stress of winding after the similarity shall be $F_{l}{ }^{\prime}=k^{4} F_{l}$.

When magnetic materials are affected by magnetic flux density, it is assumed that magnetostriction in parallel and vertical directions are, respectively:

$$
\begin{gathered}
\varepsilon_{p}=\alpha B^{2} \\
\varepsilon_{v}=-v \varepsilon_{p}
\end{gathered}
$$


According to the relationship between applied voltage and magnetic flux density:

$$
U=\frac{2 \pi}{\sqrt{2}} f N B_{S}
$$

It can be seen that the magnetostrictive force $F_{c}$ on the node is directly proportional to the square $U^{2}$ of the applied voltage, since $U^{\prime}=k U$, the magnetostrictive force on the iron core after the similarity shall be $F_{c}{ }^{\prime}=k^{2} F_{c}$.

The vibration acceleration $a_{c}$ of iron core caused by magnetostriction under the action of voltage $u$ is:

$$
a_{c}(t)=-\frac{2 \varepsilon_{s} l_{1} u^{2}}{\left(N_{1} A B_{S}\right)^{2}} \cos (2 \omega t)
$$

Among them, $\varepsilon_{S}$ is the saturated magnetostriction rate of silicon steel sheet, $\omega$ is the angular frequency of voltage source, $B_{S}$ is the saturation magnetic induction strength of iron core, $l_{1}$ is the length of silicon steel sheet and $A$ is the area of silicon steel sheet. After the model is similar, $\varepsilon_{S}$ remains unchanged, $u^{\prime}=k u, A^{\prime}=k^{2} A, B_{S}{ }^{\prime}=k B_{S}$. It can be seen that after the model is similar, the vibration acceleration on the iron core should be $a_{c}{ }^{\prime}=k^{-3} a_{c}$.

The mass spring damping system is used to analyze the axial electrodynamic force of winding. Each layer of wire cake is equivalent to mass block $m$, the insulating pad between winding wire cakes is equivalent to spring $s$, and the damping $c$ is generated by transformer oil. The differential equation of winding vibration displacement $x$ under Lorentz force $F_{l}$ is:

$$
m \ddot{x}+c \dot{x}+s x=F_{l}
$$

When the initial state is zero, the vibration acceleration is obtained:

$$
a_{l}(t)=\ddot{x}(t)=K I^{2} \cos (2 \omega t+2 \theta+\varphi)
$$

where $\varphi$ is the correlation constant of transformer parameters under initial conditions. It can be seen that the vibration acceleration of the winding is directly proportional to $I^{2}$, so the vibration acceleration of the winding after the model is similar and should be $a_{l}{ }^{\prime}=k^{4} a_{l}$. The vibration parameters before and after model similarity are listed in Table 2.

Table 2. Vibration parameters.

\begin{tabular}{ccccc}
\hline & $\begin{array}{c}\text { Lorentz } \\
\text { Force }\end{array}$ & $\begin{array}{c}\text { Magnetostrictive } \\
\text { Force }\end{array}$ & $\begin{array}{c}\text { Winding Vibration } \\
\text { Acceleration }\end{array}$ & $\begin{array}{c}\text { Core Vibration } \\
\text { Acceleration }\end{array}$ \\
\hline Original model & $F_{l}$ & $F_{c}$ & $a_{l}$ & $a_{c}$ \\
Similarity model & $k^{4} F_{l}$ & $k^{2} F_{c}$ & $k^{4} a_{l}$ & $k^{-3} a_{c}$ \\
\hline
\end{tabular}

\section{Multi Physical Field Model of Converter Transformer and Its Similarity Model}

\subsection{Physical Model of Converter Transformer}

Based on the finite element simulation platform, taking a $500 \mathrm{kV}$ converter transformer as an example, the three-dimensional physical model of the converter transformer is established. Considering the symmetrical physical structure of the converter transformer, the model is divided into four completely symmetrical parts, and only one part is studied to reduce the amount of calculation of the model. The effect of the model is shown in Figure 1. In the modeling process, the converter transformer structure is idealized, the fixtures and other structures are ignored, and the corresponding fixed constraints are imposed on the winding and iron core as the boundary conditions. The field circuit coupling method is used to couple the magnetic field model with the external circuit. The air, iron core and winding domain are given material properties. Lorentz force is applied to the winding as volume load, and a magnetostrictive effect is added to the iron core domain to study the stress on the winding and iron core, respectively. In the magnetic field physical field module, voltage source excitation is added to each winding and its input and output ports 
are set. The parameter settings of the model are shown in Table 3. In order to obtain more accurate calculation results, the tolerance is set to 0.1 , the mesh cell size is set to super refinement, the number of degrees of freedom is increased to 592,163 and the solution time is $2105 \mathrm{~s}$. The magnetic flux density distribution, stress distribution, shape variable and acceleration of each part of the converter transformer are calculated to facilitate the force and vibration mode analysis of the converter transformer.

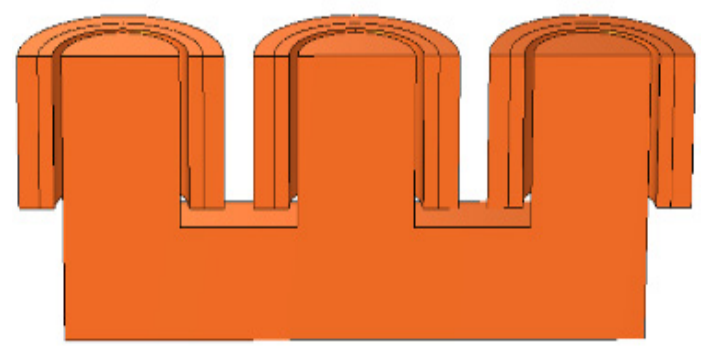

Figure 1. Simplified model.

Table 3. Model parameter configuration.

\begin{tabular}{cccc}
\hline Material Parameters & Winding & Iron Core & Air Domain \\
\hline Relative permeability & 0.98 & 8000 & 1 \\
Poisson's ratio & 0.35 & 0.45 & 0.38 \\
Young's modulus & $1.1 \times 10^{11} \mathrm{~Pa}$ & $1.2 \times 10^{11} \mathrm{~Pa}$ & $1.7 \times 10^{9} \mathrm{~Pa}$ \\
Density & $7870 \mathrm{~kg} \cdot \mathrm{m}^{-3}$ & $8960 \mathrm{~kg} \cdot \mathrm{m}^{-3}$ & $1.29 \mathrm{~kg} \cdot \mathrm{m}^{-3}$ \\
\hline
\end{tabular}

\subsection{Converter Transformer Scale Model}

According to the previous analysis of the similarity principle of a converter transformer, the corresponding parameters of the physical model of the converter transformer are similar. The similarity coefficient selected in the experiment is $k=0.1$. Accordingly, after similarity, the voltage is reduced to $k$ times the original, the resistance is increased to $k^{-1}$ times the original, the frequency is increased to $k^{-2}$ times the original, the current is reduced to $k^{2}$ times the original and the inductance and capacitance are increased to $k^{-1}$ times the original; the operating parameters of the physical model of the similar converter transformer are listed in Table 4. It should be noted that after the frequency is increased to $k^{-2}$ times, the time-related operation needs to be corrected. Set the output time in the transient research step and reduce the output time length and step size to $k^{2}$ times at the same time. The mesh cell size is also set to super refinement, the tolerance is set to 0.1 , the number of degrees of freedom is 592,163 and the solution time is $2142 \mathrm{~s}$. This paper aims to study the similarity model for the vibration analysis and suppression of a converter transformer, so the foothold of similarity model verification mainly lies in the consistency analysis of vibration before and after model similarity. The magnetic flux density distribution, stress distribution and vibration signal of the converter transformer model before and after the similarity are compared and analyzed below.

Table 4. Operating parameters.

\begin{tabular}{ccc}
\hline Parameter & Before Similarity & After Similarity \\
\hline Model width & $4 \mathrm{~m}$ & $0.4 \mathrm{~m}$ \\
Primary winding resistance & $1.68 \Omega$ & $16.8 \Omega$ \\
Secondary winding resistance & $0.2 \Omega$ & $2 \Omega$ \\
Supply voltage & $288 \mathrm{kV}$ & $28.8 \mathrm{kV}$ \\
Power voltage frequency & $50 \mathrm{~Hz}$ & $5000 \mathrm{~Hz}$ \\
Transient study scope & $0-0.05 \mathrm{~s}$ & $0-0.0005 \mathrm{~s}$ \\
Transient study step & $5.0 \times 10^{-4} \mathrm{~s}$ & $5.0 \times 10^{-6} \mathrm{~s}$ \\
\hline
\end{tabular}




\section{Results and Discussion}

\subsection{Magnetic Flux Density Distribution}

The longitudinal section of the iron core is three-dimensional symmetrical, so as to obtain the distribution diagram of magnetic flux density modulus on the iron core surface, as shown in Figure 2. The magnetic flux density values of different intensities on the iron core surface are displayed according to the legend color, and the arrow represents the direction and path of magnetic flux. It can be seen from the figure that the magnetic induction intensity distribution of the main magnetic circuit of the converter transformer before and after the similarity is basically the same, and the strong magnetic flux density points on the iron core are distributed at the iron core corner and junction, which is consistent with the previous research, that is, the magnetic flux density value at the corner is higher, and the magnetostrictive effect will be more intense. The magnetic flux density mode threshold of the model after similarity is $k$ times that before similarity, which is consistent with the magnetic field similarity condition in the similarity criterion.
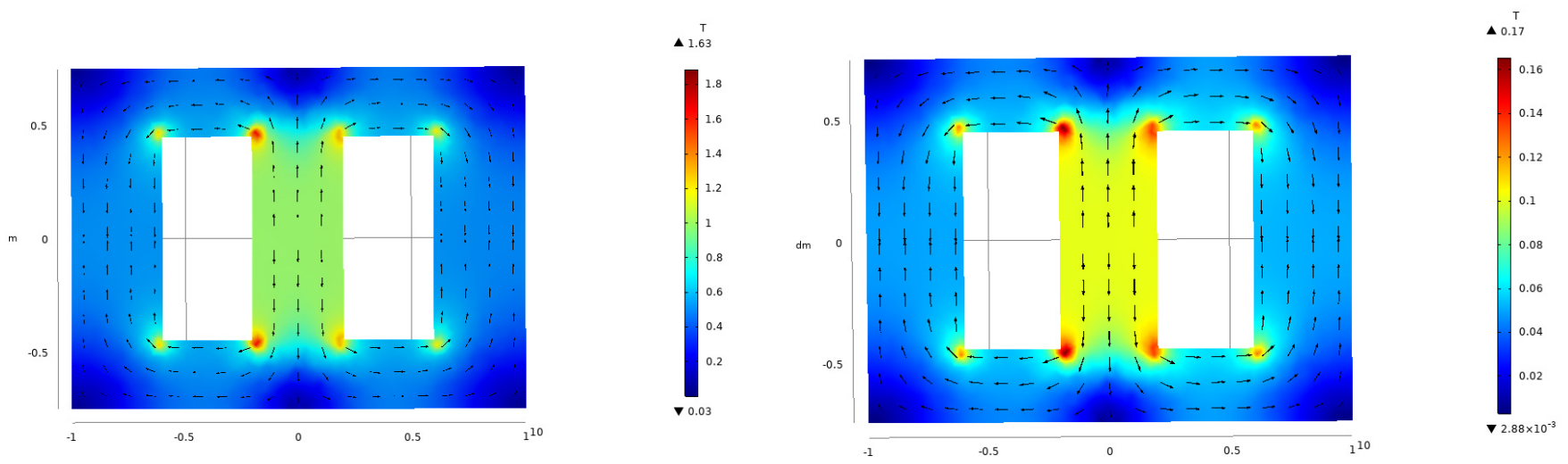

Figure 2. Magnetic flux density distribution.

\subsection{Stress Analysis}

After performing three-dimensional symmetry on the core domain and winding domain, the entire core domain and winding domain model are obtained, and the overall converter transformer domain model is obtained by the same method. Before analysis, it should be noted that the following figures show the function of finite element point stress corresponding to time. The amplitude of AC applied to the winding at different time points is different, which makes the stress at each place in the figures change with time. Since the frequency increases to 100 times after the similarity, the time point after the similarity should be 0.01 times the original time point according to the relationship between frequency and time. The time point selection of the following comparison charts is based on the above analysis.

\subsubsection{Stress Analysis of Iron Core}

Figure 3 shows the stress distribution of converter transformer core, and the red arrow indicates the direction of stress, corresponding to the legend on the right, the color on the iron core reflects the stress. It can be clearly seen from the figure that the stress distribution of iron core before and after the similarity is basically the same, which shows that the stress points on the surface of the iron core are similar before and after, and there is no change in the direction of the stress. At the same time, it can be seen that the obvious stress areas of the iron core before and after the similarity are basically the same, which are located on the magnetic flux path, and the four most obvious stress points are located at the iron core corner, which is consistent with the prediction in the previous magnetic flux density analysis. The stress amplitude of iron core before the similarity is $3.06 \times 10^{7} \mathrm{~Pa}$, the stress amplitude of the iron core after the similarity is $3.47 \times 10^{5} \mathrm{~Pa}$, the relationship between them is approximately $k^{2}$ times. The relationship curve between magnetostrictive length 
and magnetic flux density can be approximately expressed as a quadratic relationship. According to the formula $U=2 \pi / \sqrt{2} f N B_{s}$, the magnetostrictive force on the iron core is directly proportional to the square of voltage. Since the vibration of the iron core mainly depends on the magnetostrictive effect, the stress size and distribution shown in the figure are reasonable $[15,16]$.
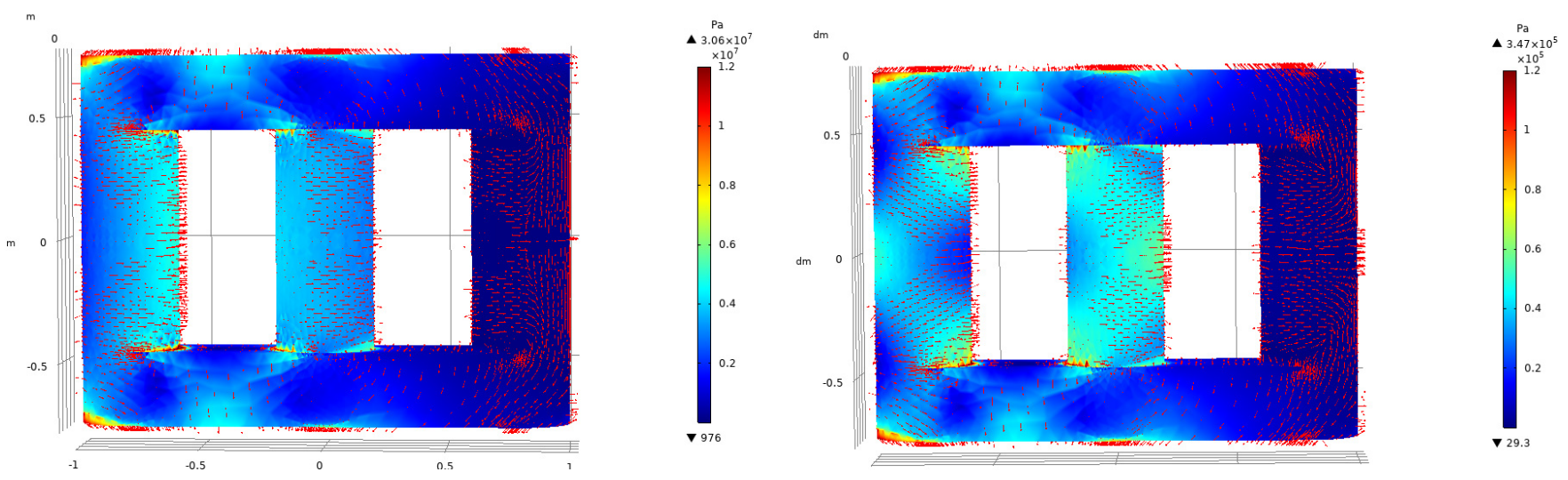

Figure 3. Stress distribution of iron core.

\subsubsection{Winding Stress Analysis}

Figure 4 shows the stress distribution of converter transformer winding. It can be seen that the stress points, stress directions and stress distribution of windings before and after the similarity are consistent. The stress amplitude of similar front winding is $363 \mathrm{~Pa}$ and that of similar rear winding is $0.04 \mathrm{~Pa}$, showing an approximate $k^{4}$ relationship, which is consistent with the analysis of formula (5) for calculating the electric power on the winding. Due to the relatively low hardness of winding material, the change of stress with current not only depends on electromagnetic force, but also is related to the vibration of iron core and other parts and the periodic change of electric field. There is a certain error between the change of value before and after the similarity and the expected proportional relationship, but the corresponding relationship is maintained in the order of magnitude.
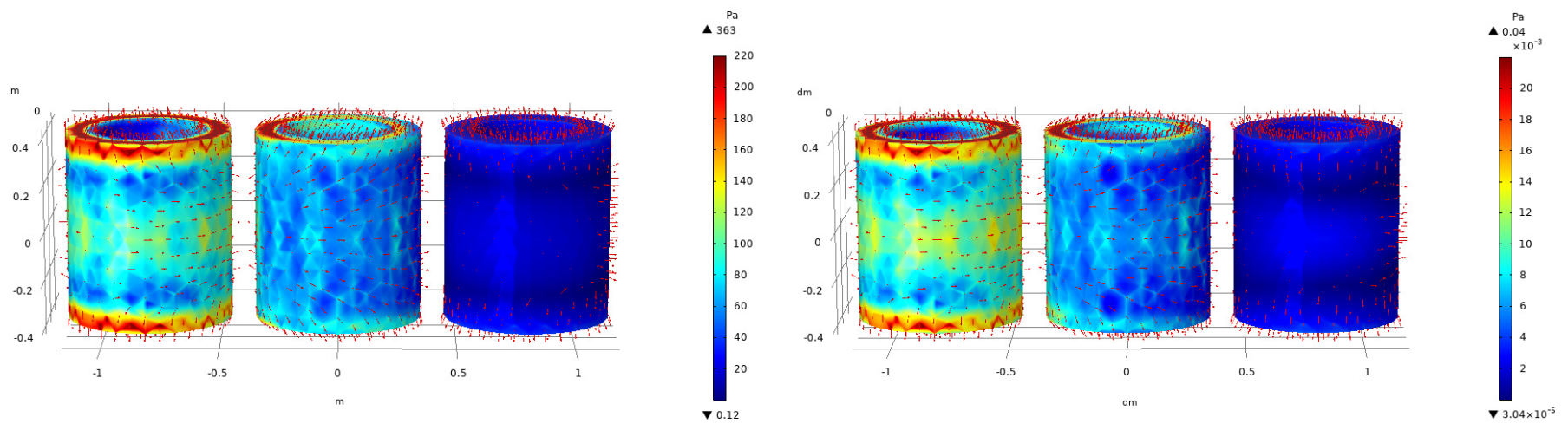

Figure 4. Winding stress distribution.

\subsubsection{Overall Stress Analysis of Converter Transformer}

Figure 5 shows the overall stress of converter transformer. It can be seen from the figure that the surface stress points, stress direction and surface stress distribution are basically the same before and after the similarity. It can be seen that the overall stress threshold of the converter transformer is very close to that of the iron core, while the stress of winding is not obvious in the overall stress distribution. Compared with the stress on the iron core, they are quite different in order of magnitude. As previously analyzed, the overall stress of the converter transformer is dominated by the iron core, that is, the biggest 
factor affecting the vibration of the converter transformer is the magnetostriction effect of the iron core.
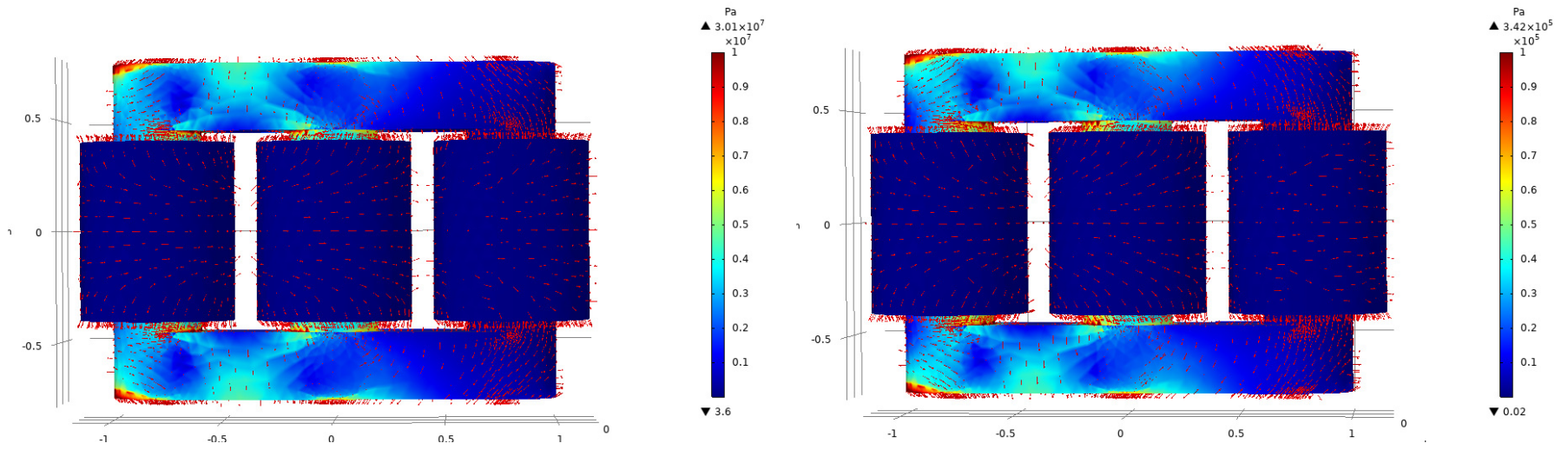

Figure 5. Overall stress distribution.

\subsection{Deformation Analysis of Winding and Iron Core}

As shown in Figure 6, the deformation trend and deformation distribution of windings before and after the similarity are consistent, and the amplitude of winding shape variable before the similarity is $3.43 \times 10^{-10}$, the amplitude of winding shape variable after the similarity is $3.82 \times 10^{-14}$, the difference is about $k^{4}$ times. The winding deformation mainly comes from the electromagnetic force generated by current and magnetic flux leakage, and its variation law is consistent with the similar process of current. Figure 7 shows the deformation of the converter transformer core. The deformation shape and shape variable distribution of the iron core before and after the similarity are consistent, and the difference of shape variable threshold before and after the similarity is $k^{2}$ times. With the continuous progress of transformer production technology, the gap between iron core silicon steel sheets is becoming smaller and smaller, so the electromagnetic force between laminations can no longer be considered. Therefore, the deformation of the iron core mainly comes from magnetostriction under the magnetic field, which is directly proportional to the square of voltage. In conclusion, the deformation law of the converter transformer is consistent before and after the similarity.
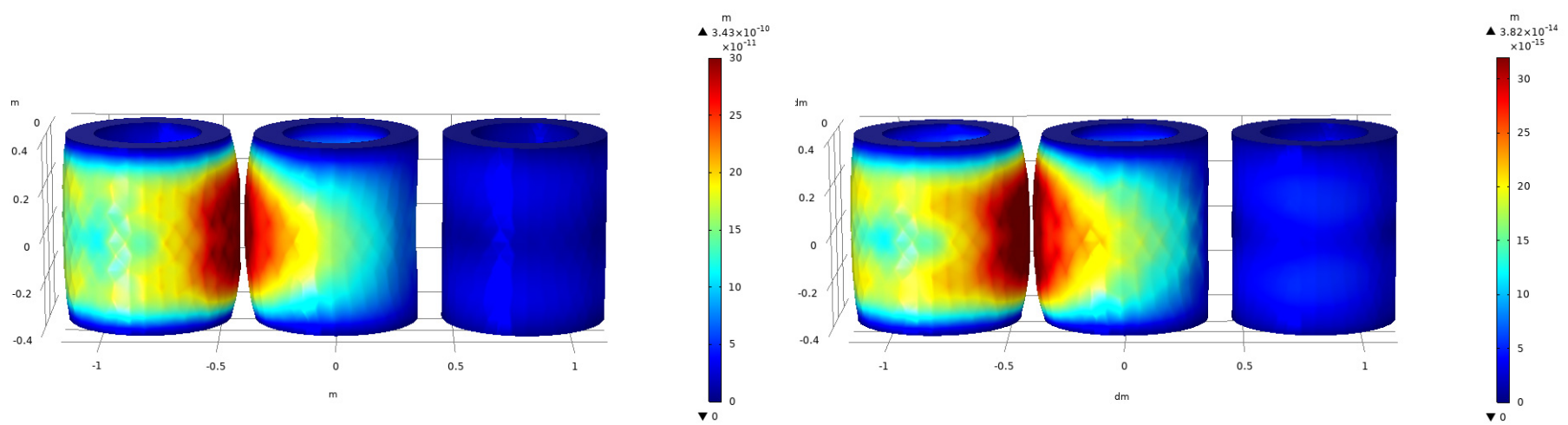

Figure 6. Winding displacement. 

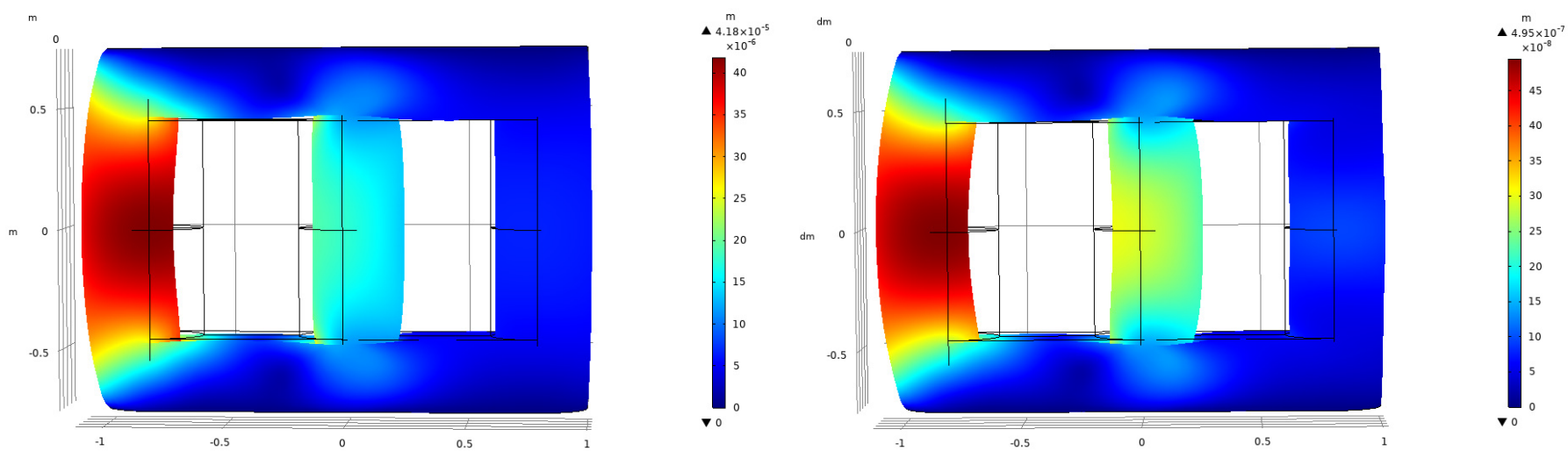

Figure 7. Core displacement.

\subsection{Vibration Signal Analysis}

A point on the surface of the iron and core winding was taken for vibration signal extraction, named point 1 and point 2, respectively, as shown in Figure 8. Figure 9a,d, respectively, show the vibration acceleration spectrum at point 1 before and after the similarity. It can be seen that the maximum peak values of the vibration acceleration before and after the similarity are distributed at $100 \mathrm{~Hz}$ and $10,000 \mathrm{~Hz}$, respectively, which corresponds to the double relationship with the frequency values of their respective excitation sources. The difference is that the maximum peak value of vibration acceleration of the iron core after the similarity is $191.26 \mathrm{~m} / \mathrm{s}^{2}$, which is increased compared with the maximum peak value of vibration acceleration before the similarity of $0.18 \mathrm{~m} / \mathrm{s}^{2}$, which is approximately $k^{-3}$ times, which is consistent with the analysis of formula (9). Figure $9 \mathrm{~b}$,e, respectively, correspond to the stress time curve at point 1 before and after the similarity. The waveforms of the two are almost the same, and the peaks of the curves differ by approximately $k^{2}$ times. Figure $9 c, d$, respectively, characterize the magnetostrictive contribution at point 1 before and after the similarity. It can be seen that the magnetostrictive force time waveform at this point before and after the similarity is consistent, and the peak values of the two are also $k^{2}$ times different. It should be noted that the magnetostrictive contribution at point 1 is close to the stress value at this point before and after the similarity, that is, before and after the similarity of the model, the stress on the iron core depends on its magnetostrictive effect under the magnetic field.

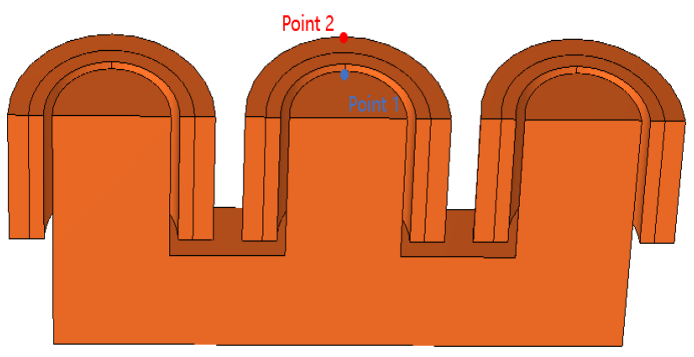

Figure 8. Vibration signal extraction points. 

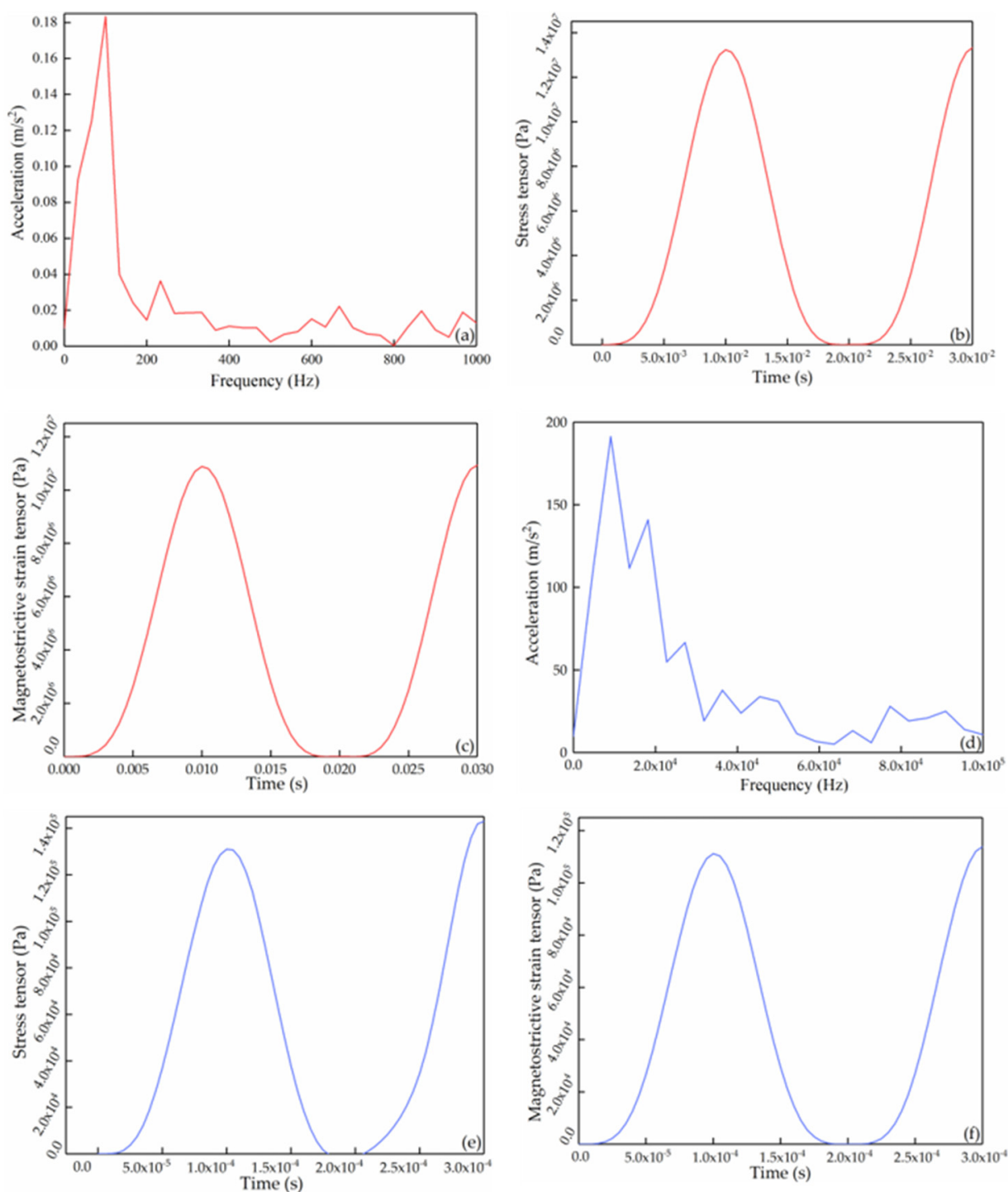

Figure 9. Vibration signal of point 1 before and after similarity. (a) acceleration before similarity, (b) stress before similarity, (c) magnetostrictive contribution before similarity, (d) acceleration after similarity (e) stress after similarity (f) magnetostrictive contribution after similarity.

Figure 10 shows the vibration signal analysis diagram at the winding signal extraction point. Figure 10a,d show the vibration acceleration spectrum at point 2 before and after the similarity. The maximum peak values of vibration acceleration before and after the similarity are also distributed at $100 \mathrm{~Hz}$ and $10,000 \mathrm{~Hz}$, respectively, and the amplitude of the similar front winding shape variable is $3.67 \times 10^{-2} \mathrm{~m} / \mathrm{s}^{2}$ and that of the similar rear winding shape variable is $3.42 \times 10^{-6} \mathrm{~m} / \mathrm{s}^{2}$, which is consistent with the analysis of formula (11). Figure 10b,e, respectively, correspond to the stress change curves at point 2 before and after the similarity. The waveforms of the two are almost the same, and the peak values of the curves are about $k^{4}$ times different. Figure 10c,d, respectively, represent the Lorentz force contribution of point 2 before and after the similarity. The waveform shapes of the two are almost the same, and the peak values are $k^{4}$ times different, this is consistent with the stress distribution of the winding. 

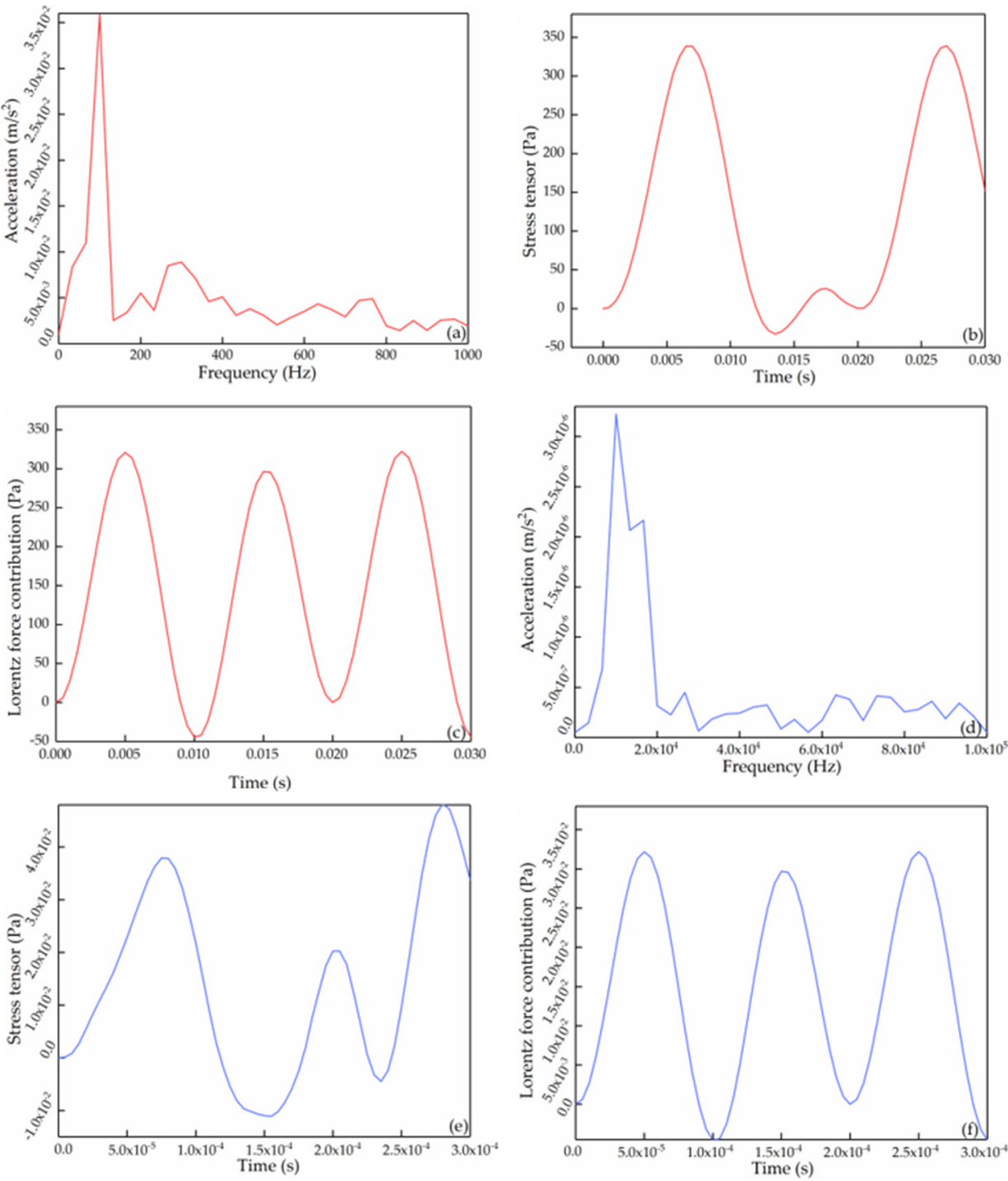

Figure 10. Vibration signal of point 2 before and after similarity. (a) acceleration before similarity, (b) stress before similarity, (c) magnetostrictive contribution before similarity, (d) acceleration after similarity (e) stress after similarity (f) Lorentz force contribution after similarity.

\subsection{Vibration Signal Restoration}

As analyzed above, the magnetic flux density threshold of the proportional model is $k$ times before the similarity, the overall stress of the iron core and converter transformer is $k^{2}$ times before the similarity, and the winding stress is $k^{4}$ times before the similarity. When the proportional model is established and its vibration is analyzed, the signal measured by the proportional model is multiplied by the corresponding coefficient to restore the signal value of the actual converter transformer, that is, to ensure that the response relationship between the input and vibration of the similar front and rear converter is unchanged. This step meets the idea of "point symmetry" described in the introduction.

\section{Conclusions}

Through the comparative analysis of magnetic flux density distribution, stress distribution, shape variable and vibration time-domain and frequency-domain signals, it is proved that the model of converter transformer before and after the similarity corresponds to the similarity criterion in electromagnetic field and structural mechanics, so as to comprehensively verify the correctness of the similarity model of the converter transformer. The comparison results show that the main vibration of the similar model of the 
converter transformer is still the magnetostrictive effect of iron core under the magnetic field, and the main frequency of the vibration signal is even times of the excitation source. The idea of "point symmetry" embodied in the similarity and reduction of the converter transformer vibration model in this paper has a certain reference value for the analysis of other scientific problems.

Author Contributions: Conceptualization, L.Z. (Li Zhang) and L.Z. (Liang Zou); methodology, G.W.; software, H.W.; validation, H.W.; formal analysis, Y.S.; investigation, Y.S.; resources, Y.S.; data curation, L.Z. (Li Zhang); writing—original draft preparation, H.W.; writing-review and editing, H.W.; visualization, H.W.; supervision, L.Z. (Li Zhang); project administration, L.Z. (Li Zhang); funding acquisition, Y.S. All authors have read and agreed to the published version of the manuscript.

Funding: Science and Technology Project of State Grid Corporation (5500-202124103A-0-0-00).

Institutional Review Board Statement: Not applicable.

Informed Consent Statement: Not applicable.

Acknowledgments: This work was supported by the Science and Technology Project of State Grid Corporation. Research on key technology of suppressing vibration and reducing noise of converter transformers (5500-202124103A-0-0-00).

Conflicts of Interest: The authors declare no conflict of interest.

\section{References}

1. Hammons, T.; Woodford, D.; Loughtan, J.; Chamia, M.; Donahoe, J.; Povh, D.; Bisewski, B.; Long, W. Role of HVDC transmission in future energy development. IEEE Power Eng. Rev. 2000, 20, 10-25. [CrossRef]

2. Zhao, Y.; Crossley, P. Impact of DC bias on differential protection of converter transformers. Int. J. Electr. Power Energy Syst. 2020, 115, 105426. [CrossRef]

3. Liu, X.; Wu, J.; Jiang, F.; Wang, Y.; Zhang, C.; Hui, Y. Electromagneto-mechanical numerical analysis and experiment of transformer influenced by DC bias considering core magnetostriction. J. Mater. Sci. Mater. Electron. 2020, 31, 16420-16428. [CrossRef]

4. Weiser, B.; Pfützner, H.; Anger, J. Relevance of magnetostriction and forces for the generation of audible noise of transformer cores. IEEE Trans. Magn. 2000, 36, 3759-3777. [CrossRef]

5. Weiser, B.; Hasenzagl, A.; Booth, T.; Pfützner, H. Mechanisms of noise generation of model transformer cores. J. Magn. Magn. Mater. 1996, 160, 207-209. [CrossRef]

6. Kitagawa, W.; Ishihara, Y.; Todaka, T.; Nakasaka, A. Analysis of structural deformation and vibration of a transformer core by using magnetic property of magnetostriction. Electr. Eng. Jpn. 2010, 172, 19-26. [CrossRef]

7. Li, Q.; Wang, X.; Zhang, L.; Lou, J.; Zou, L. Modelling methodology for transformer core vibrations based on the magnetostrictive properties. IET Electr. Power Appl. 2012, 6, 604-610. [CrossRef]

8. $\quad \mathrm{Pu}, \mathrm{Z} .-\mathrm{H} . ;$ Ruan, J.-J.; Du, Z.-Y.; Zhang, Y.-D.; Li, J.-L.; Xie, Q.-J.; Tan, D. Analysis of Voltage Distribution Characteristics in UHVDC Converter Transformer Winding Based on the Reduced-Scale Model. IEEE Trans. Magn. 2014, 50, 1-5. [CrossRef]

9. Zhang, P.; Li, L.; Cheng, Z.; Tian, C.; Han, Y. Study on Vibration of Iron Core of Transformer and Reactor Based on Maxwell Stress and Anisotropic Magnetostriction. IEEE Trans. Magn. 2018, 55, 1-5. [CrossRef]

10. Moses, A.J.; Anderson, P.I.; Phophongviwat, T. Localized Surface Vibration and Acoustic Noise Emitted from Laboratory-Scale Transformer Cores Assembled from Grain-Oriented Electrical Steel. IEEE Trans. Magn. 2016, 52, 1-15. [CrossRef]

11. Sayed, A.M.; Abouelatta, M.A.; Badawi, M.; Mahmoud, K.; Lehtonenm, M.; Darwish, M.M.F. Novel accurate modeling of dust loaded wire-duct precipitators using FDM-FMG method on one fine computational domains. Electr. Power Syst. Res. 2022, 203, 107634. [CrossRef]

12. Zalhaf, A.S.; Mansour, D.-E.A.; Han, Y.; Yang, P.; Yang, P.; Darwish, M.M.F. Numerical and Experimental Analysis of the Transient Behavior of Wind Turbines when Two Blades are Simultaneously Struck by Lightning. IEEE Trans. Instrum. Meas. 2021. [CrossRef]

13. Abouelatta, M.A.; Ward, S.A.; Sayed, A.M.; Mahmoud, K.; Lehtonen, M.; Darwish, M.M.F. Measurement and assessment of corona current density for HVDC bundle conductors by FDM integrated with full multigrid technique. Electr. Power Syst. Res. 2021, 199, 107370. [CrossRef]

14. Abouelatta, M.A.; Ward, S.A.; Sayed, A.M.; Mahmoud, K.; Lehtonen, M.; Darwish, M.M.F. Fast Corona Discharge Assessment Using FDM integrated With Full Multigrid Method in HVDC Transmission Lines Considering Wind Impact. IEEE Access 2020, 8, 225872-225883. [CrossRef]

15. Garcia, B.; Burgos, J.C.; Alonso, A. Transformer Tank Vibration Modeling as a Method of Detecting Winding Deformations-Part I: Theoretical Foundation. IEEE Trans. Power Deliv. 2006, 21, 157-163. [CrossRef]

16. Garcia, B.; Burgos, J.C.; Alonso, A. Transformer Tank Vibration Modeling as a Method of Detecting Winding Deformations-Part II: Experimental Verification. IEEE Trans. Power Deliv. 2006, 21, 164-169. [CrossRef] 